\title{
Chercher ensemble : les défis et les cahots de l'interdisciplinarité, des méthodes mixtes et des partenariats multiples (Rume)
}

\author{
Isabelle Guérin \\ Emmanuelle Bouquet \\ Solène Morvant-Roux
}

MOTS-CLÉS

Projet de recherche, méthodologie, interdisciplinarité, partenariat.

\section{RÉSUMÉ}

Cet article décrit la construction et le déroulement d'un projet de recherche interdisciplinaire (économie/anthropologie), multi-méthodes (quantitatif et qualitatif) et multi-partenarial (Inde, Mexique, Madagascar) afin d'en tirer des enseignements en matière de modes de production de connaissance. Les clivages épistémologiques (quel réel observet-on et comment valide-t-on les connaissances ainsi produites ?) et les confusions dans l'usage des termes "quantitatif ", " qualitatif " et " partenariat » ont été des points d'achoppement récurrents. Le projet a néanmoins produit des résultats novateurs, mais au prix d'un processus cahotant d'adaptation, de créativité et de compromis, à la fois sur le plan scientifique, partenarial, et humain. 



\section{Introduction}

Tnterdisciplinarité, pluralisme méthodologique et partenariats : ce sont là Ltrois mots-clés dans les recherches actuelles menées sur les Suds. Combiner et décloisonner les regards disciplinaires et les outils d'analyse apparaît comme une nécessité sur de nombreuses questions de développement. Simultanément, et suite à plusieurs décennies de " coopération » scientifique souvent réduite à des relations avec des chercheurs du Sud isolés, cantonnés au rôle d'informateurs cléfs, de guides locaux, de clients ou de protégés, il semble indispensable de construire des partenariats dignes de ce nom, c'est-à-dire reposant sur des rapports de force équilibrés (Copans, 2010).

S'il s'agit là d'objectifs sensés et louables, leur mise en pratique est loin d'aller de soi, a fortiori dans un contexte caractérisé par le durcissement des cloisonnements disciplinaires. S'appuyant sur un projet portant sur la finance rurale (Rural microfinance and employment: Do processes matter?), financé par l'Agence nationale de la recherche, et qui s'est déroulé entre 2008 et 2011, cet article est un effort de réflexivité sur la mise en œuvre concrète d'une recherche interdisciplinaire (avec ici un dialogue entre l'économie et l'anthropologie), multi-méthodes (qualitatif et quantitatif) et partenarial (le partenariat étant envisagé à la fois comme une collaboration entre équipes « Nord » et équipes «Sud », et comme une collaboration entre chercheurs statutaires et chercheurs contractuels - les post-doctorants). Il résume brièvement les objectifs et le déroulement du projet. Sur la base du vécu et du point de vue (nécessairement partial) des trois auteures, l'article décrit ensuite les différents acquis et obstacles, tout d'abord ceux concernant la rencontre entre disciplines et entre méthodes, puis ceux liés à la recherche en partenariat. Les clivages épistémologiques (quel réel observe-t-on et comment valide-t-on les connaissances ainsi produites ?) et les confusions, pour partie liées au point précédent, dans l'usage des termes « quantitatif », « qualitatif » mais aussi " partenariat», ont été des points d'achoppement récurrents. La dernière section propose quelques enseignements en matière de modes collectifs de production de connaissances : quelles sont les significations et les implications de « chercher ensemble »? En définitive, le projet a produit des résultats novateurs, et valorisés dans la sphère académique, mais au prix d'un processus cahotant d'adaptation, de créativité et de compromis, à la fois sur le plan scientifique, partenarial, et humain. 


\section{Le projet : objectifs et dispositifs}

Cette recherche avait pour objectif d'étudier les interactions entre finance rurale (notamment la microfinance, mais aussi l'ensemble des pratiques financières que les familles mobilisent au quotidien) et emploi dans trois pays ou sous-régions : l'Inde du Sud, le Mexique et Madagascar. Conçu au départ par trois économistes/socio-économistes françaises (les trois auteures de l'article), ce projet avait pour spécificité première d'impliquer des anthropologues. Nous étions convaincues que l'inscription sociale et politique des objets étudiés était fondamentale, et même si nos cadres théoriques d'analyse (socio-économie, économie néo-institutionnelle) leur accordaient déjà une certaine importance, nous souhaitions aller plus loin en enrichissant notre approche d'un regard anthropologique. En termes de méthode, et toujours dans le prolongement de nos travaux antérieurs, nous envisagions de coupler plusieurs outils de collecte et d'analyse, pratique communément qualifiée de méthode mixte «quantitatif/qualitatif ». Celle-ci postule que les forces du quantitatif peuvent potentiellement compenser les faiblesses du qualitatif et vice versa (Rao et Woolcock, 2003, p. 168). Il allait enfin de soi qu'il nous fallait impliquer des collègues des trois pays étudiés.

L'ambition de " chercher ensemble », en associant des chercheurs de différentes disciplines et de différents pays, s'articulait autour de deux enjeux. Le premier consistait à construire un objet commun puis une méthode commune. Alors que l'interdisciplinarité prend le plus souvent la forme d'un regard croisé sur un même objet, nous misions sur le fait qu'il était à la fois nécessaire, possible et fructueux sur le plan heuristique d'associer les deux disciplines. Le deuxième enjeu portait sur la production de données empiriques pertinentes, fiables et mobilisables dans une démarche multi-méthodes. Nous avions prévu de capitaliser sur des données collectées antérieurement sur les trois terrains, mais aussi et surtout de collecter des matériaux empiriques nouveaux, quantitatifs (via des enquêtes ménages à grande échelle) et qualitatifs (via différents outils d'observation directe, d'observation participante, d'entretiens approfondis), et pensés conjointement entre disciplines et aires géographiques ${ }^{1}$.

1 Le projet affichait également une ambition comparative entre les trois terrains d'étude. La production des données a été pensée dans cet objectif, et les corpus empiriques produits dans le cadre du projet permettent effectivement de mener 
La composition de l'équipe du projet, en traduisant ces différentes préoccupations, était porteuse à la fois de diversités et de proximités. L'équipe rassemblait des profils divers, que ce soit par leur ancrage disciplinaire, leur parcours professionnel, leur pays, leur institution de rattachement, et leur statut (permanent ou contractuel). Cette diversité faisait courir le risque de difficultés dans la communication, la compréhension mutuelle, les échanges et la production scientifique. Dans le même temps, des facteurs de rapprochement laissaient présager la possibilité d'espaces de travail commun. Les économistes s'inscrivaient (à des degrés divers) dans des courants hétérodoxes. Ils partageaient avec les anthropologues un intérêt pour les approches compréhensives et processuelles, ainsi qu'une vigilance sur la question du rapport au terrain et la production de données empiriques de qualité.

Au moment de la clôture officielle du projet, en avril 2011, le bilan est très honorable selon les canons d'évaluation de l'ANR. Un corpus empirique considérable a été constitué, combinant effectivement des matériaux quantitatifs et qualitatifs. La production académique, amorcée en 2011 et qui a continué de s'étoffer depuis, est conséquente ${ }^{2}$. Des résultats novateurs ont été produits, concernant notamment la diversité des effets de la microfinance, les manifestations et les implications du surendettement, l'articulation des pratiques financières formelles et informelles (Guérin, Morvant-Roux, Villarreal (dir.), 2013). Des concepts tels que le jonglage financier (Roesch, Héliès, 2007) ou les cadres de calcul (Villarreal, 2009) ont circulé entre chercheurs de différentes traditions, alimentant des réflexions croisées et permettant de revisiter certaines questions (Wampfler, Bouquet, Ralison, 2013 ; Picherit, 2013).

Néanmoins, cette ambition de « chercher ensemble » a posé de multiples défis. Nous en avions anticipé certains, mais pas tous, et les défis anticipés avaient été clairement sous-estimés. Les acquis du projet ont ainsi

à bien cette démarche comparative. Néanmoins, l'exercice de comparaison à proprement parler n'avait pas encore débuté au moment de la rédaction de cet article, et il n'en sera par conséquent pas question dans ce texte.

2 Guérin, Lapenu et Doligez (dir.), 2009 ; Guérin, Roesch, Michiels et Venkatasubramanian, 2012 ; Guérin, Morvant-Roux et Villarreal (dir.), 2013 ; Angulo et Villarreal (dir.), 2013 ; Villarreal (dir.), 2014 ; Bouquet, Morvant-Roux et Rodriguez-Solis, à paraître ; Guérin, D’Espallier et Venkatasubramanian, à paraître. 
émergé d'un processus particulièrement laborieux et chronophage. La suite de l'article se propose d'explorer quelques aspects qui nous semblent porteurs d'enseignements à vocation plus générale pour la recherche sur le développement au Sud.

\section{La vie scientifique d'un projet : portée et défis de I'interdisciplinarité et du croisement des méthodes}

\subsection{Construire un questionnement commun}

La première étape a consisté à construire collectivement un but commun. Même si le projet contenait dès sa formulation initiale un certain nombre de questions, il nous semblait essentiel que l'ensemble de l'équipe, et notamment les anthropologues, se réapproprie et reformule à sa manière les objectifs du projet. Il a fallu tout d'abord un certain temps pour identifier les objectifs de notre démarche interdisciplinaire, qui seront in fine formulés de la manière suivante : pour les économistes, quelles sont les logiques et pratiques, négligées ou occultées par l'approche économique, que l'anthropologie peut nous aider à révéler, qui fragilisent l'analyse et qui méritent d'être analysées et éventuellement quantifiées par rapport à la question de recherche posée ? Pour les anthropologues, quels sont les éléments de leurs analyses qu'il est à la fois souhaitable et possible de quantifier et de systématiser?

Sur cette base, nous avons abouti à la question suivante : étudier la diversité des relations financières, des relations d'emploi et des relations sociales qui leur sont sous-jacentes ainsi que leurs interactions. Si cette question était finalement très proche de notre question initiale, ce processus de réappropriation collective, qui nous semblait incontournable, nous aura pris près d'un an et demi.

\subsection{Articuler qualitatif et quantitatif : une question de méthode et d'épistémologie}

Le cœur de notre projet consistait à combiner les méthodes, qualifiées dans le document initial de «quantitatives » et « qualitatives » en les mobilisant non pas de manière séquentielle ou parallèle mais itérative, tout 
au long du projet. Une première vague de « qualitatif » visait à poser des hypothèses novatrices et construire une enquête par questionnaire adaptée au contexte local; dans un second temps, l'analyse statistique avait pour but de quantifier et de mettre en évidence des corrélations; enfin, dans un troisième temps, une nouvelle vague de « qualitatif » devait permettre d'interpréter les analyses statistiques (Rao et Woolcock, 2003, p. 172-18o).

Tous les membres de l'équipe avaient une pratique confirmée de « travail de terrain ». Si ce point est évident pour les anthropologues, il l'est beaucoup moins en économie. Or tous les économistes du groupe avaient pour particularité de collecter eux-mêmes leurs données, et d'accorder une importance centrale à la fiabilité des informations collectées. Leur définition de la fiabilité des données se rapprochait également des préoccupations des anthropologues, notamment à travers la nécessité de tenir compte des catégories « indigènes », et de la pluralité des logiques d'acteurs. L'intérêt commun pour la notion de processus, qui était au cœur du projet, doit également être noté. Si cela n’a rien d'étonnant pour les anthropologues, appréhender les personnes ainsi que leurs pratiques comme le fruit d'interactions et d'histoires collectives est beaucoup moins courant chez les économistes, qui ont tendance à se focaliser sur les résultats des actions menées. Les économistes partageaient également la vision des anthropologues sur l'importance d'une démarche itérative entre terrain et question de recherche, cette dernière devant pouvoir se prêter à des reformulations pour gagner en pertinence empirique.

Malgré cet intérêt commun pour le terrain et le pluralisme méthodologique, des clivages ont surgi entre les membres de l'équipe. Ces clivages ne portaient pas tant sur l'intérêt intrinsèque du pluralisme méthodologique, mais plutôt sur une série de questions surplombantes. Un premier aspect tournait autour de ce qui constitue une donnée scientifique observable, fiable, mobilisable pour l'analyse, et sur les moyens à déployer pour obtenir ce type de données. Il s'est accompagné de malentendus récurrents sur l'usage des termes « quantitatif » et « qualitatif ». Ces termes peuvent s'appliquer à des variables, des outils de collecte de données, des modes d'analyse, voire à des disciplines (Beaud et Weber, 2003 ; King et al., 1994) et notre projet n’a pas échappé à cette confusion. Dans l'esprit des différents membres de l'équipe, le terme « quantitatif » désignait tantôt des enquêtes 
par questionnaires, le recours à l'analyse statistique ou tout simplement le comptage. Le terme « qualitatif » signifiait tantôt des entretiens approfondis, mais visant la collecte d'information, systématisés auprès d'individus ou de ménages, tantôt de l'observation participante ou de l'observation de longue durée ciblée sur des systèmes de relations et d'interactions. Un deuxième point fondamental, et lié au précédent, mais finalement moins discuté au sein du projet, portait sur les divergences dans les modalités d'administration de la preuve. En filigrane, se posait la question des possibilités concrètes de dialogue autour de ces différents outils et entre les traditions de recherche qui s'y rattachent.

Les difficultés que nous avons rencontrées rejoignent celles d'autres équipes engagées dans une démarche similaire. En introduction d'un numéro spécial de la revue World Development consacré aux approches mixtes, Ravi Kanbur et Paul Shaffer (2007) font le constat d'un certain consensus quant à la nécessité des approches mixtes, mais soulignent que leur concrétisation pose des défis multiples et souvent négligés, du fait de clivages de nature épistémologique. Un clivage épistémologique fondamental concerne les modes d'administration de la preuve, avec d'un côté des approches que l'on pourrait qualifier de statistiques/inférentielles et les approches que l'on pourrait qualifier d'interprétatives et de compréhensives (Couty et Winter, 1983 ; King et al., 1994 ; Bardhan et Ray, 2008). Ces deux types d'approches renvoient à deux constellations de pratiques de recherche. Les premières procèdent par extrapolation, par simplification, et par test d'hypothèses. Elles requièrent des observations systématiques sur un ensemble d'unités d'analyse comparables ${ }^{3}$. Les secondes visent à mettre à jour des modes de fonctionnement et de reproduction d'entités sociales complexes, les interactions multiples, dynamiques, contradictoires entre différentes entités, tout en accordant une attention centrale aux points de vue des acteurs et aux systèmes de significations qui sous-tendent leurs pratiques (les discours n'ayant de sens qu'en lien avec le contexte de leur formulation). Ce deuxième type d'approche a pour objectif premier de produire de l'intelligibilité, pas de la généralisation. Ce clivage en appelle

3 En première analyse, cela correspond typiquement aux enquêtes quantitatives sur grands échantillons. Néanmoins, voir King et al., (1994) pour un plaidoyer en faveur d'une démarche inférentielle, y compris dans les approches qualitatives et les petits échantillons. 
d'autres. La réalité est-elle objective et observable (ce qui n'empêche pas de penser sa complexité) ou bien n'existe-t-il que des points de vue multiples, qui coexistent, interagissent et se transforment? Quelle est l'unité d'observation pertinente (individus et ménages ou systèmes de relations et d'interactions) ? Les comportements économiques sont-ils contraints par les normes sociales ou bien sont-ils des comportements sociaux? Etc.

Ces deux pratiques de recherche renvoient schématiquement au clivage quantitatif/qualitatif, ainsi qu'aux traditions de recherche de l'économie et de l'anthropologie, respectivement, sans toutefois que cette superposition soit complète. Par exemple la socio-économie se rapproche beaucoup plus de l'approche anthropologique qu'économique.

\subsection{Interdisciplinarité et multi-méthodes en pratique}

Les économistes du projet avaient une expérience dans la mise en œuvre d'outils de collecte de données qualitatifs, essentiellement à travers les entretiens approfondis permettant d'expliciter des éléments de contexte d'une part, de mettre en regard les pratiques et les discours sur les pratiques d'autre part. Cette pratique des entretiens approfondis rapprochait les économistes de certains anthropologues du projet (même si le contenu des entretiens et les formes de mobilisation des données restaient assez distincts, et ont pu être sources de tension, comme on le verra plus loin). En revanche, elle constituait une source de clivage avec une autre posture qualitative, plus proche de l'ethnographie, qui privilégie l'observation directe de longue durée, considérée comme seule à même de produire des données fiables concernant les pratiques en train de se faire (par opposition à un entretien qui se contente de les reconstituer ex post par le discours). Ce deuxième type de posture était par nature beaucoup moins propice à un travail commun, y compris entre anthropologues. La mise en œuvre de ce type de travail de terrain impliquait une immersion sur un temps long, moins compatible avec le chronogramme du projet, et compliquant la possibilité de regards croisés avec d'autres terrains.

Par ailleurs, les anthropologues ont à plusieurs reprises manifesté de la défiance vis-à-vis de la démarche qualitative des économistes : les références à l'anthropologie économique et la mobilisation d'outils semblables aux 
leurs (entretiens approfondis en l'occurrence), mais analysés sous des angles différents, et parfois de manière plus légère (par exemple, dans un objectif de description du contexte ou de restitution de logiques d'action), ont rencontré peu d'adhésion. Il est probable qu'elles aient plutôt prêté le flanc à des critiques de superficialité ou d'utilisation opportuniste d'un vernis anthropologique censé fournir un alibi de pertinence empirique. En revanche, la mobilisation de matériaux qualitatifs pour construire conjointement des catégories d'analyse pertinentes et injectables dans le questionnaire quantitatif a suscité davantage d'intérêt. Le fait que le questionnaire quantitatif soit essentiellement basé sur des variables catégorielles a probablement simplifié les choses, en permettant de laisser de côté les débats sur les questions de mesure et de commensurabilité, qui figurent souvent en premier plan dans l'exposition des critiques croisées entre méthodes. La contribution des anthropologues à la construction du questionnaire quantitatif s'est néanmoins accompagnée d'un certain scepticisme sur la capacité du réel à se plier à un système de classification, si sophistiqué et contingent soit-il.

Lorsque les anthropologues ont été impliqués dans l'élaboration du questionnaire quantitatif, leur méconnaissance de certains impératifs méthodologiques et techniques a suscité d'autres types d'incompréhensions. Pourquoi multiplier les questions sur une même thématique (or ceci est indispensable au recoupement de l'information, exactement comme dans le qualitatif) ? Pourquoi introduire de nombreuses questions a priori déconnectées du sujet (mais indispensables comme variables de contrôle pour mener des analyses multivariées) ? Ces interpellations sur la longueur du questionnaire renvoyaient à une préoccupation plus large des anthropologues sur la violence implicite qu'implique l'application de ce type d'outil d'enquête : violence conceptuelle lorsque le cadre est trop rigide, violence relationnelle, a fortiori en milieu rural dans les pays en développement, lorsque la relation entre enquêteur et enquêté est asymétrique. Cette préoccupation vis-à-vis de la violence renvoyait à des considérations éthiques, mais aussi méthodologiques, car il est fondé de s'interroger sur la qualité des données produites dans un tel contexte.

Le positionnement des anthropologues pouvait ainsi mener à remettre en question l'utilisation même de l'outil d'enquête quantitative. Bien que conscientes de ces risques, les économistes étaient sur un positionnement 
différent : elles partaient de la nécessité d’appliquer cet outil, indispensable compte tenu de leurs objectifs de recherche, et d'en respecter la logique en se conformant à ses exigences techniques, tout en cherchant à en atténuer les effets pervers.

Inversement, si l'on prend l'exemple du terrain mexicain, des économistes ont dans un premier temps été désarçonnées par le caractère non systématique du corpus d'entretiens approfondis rassemblé par certains anthropologues, qui était pourtant d'une grande finesse et se prêtait sans peine à une analyse interprétative. Elles ont néanmoins pu en tirer parti en extrayant certains thèmes ou pratiques qui semblaient particulièrement pertinents dans le contexte considéré, et en les injectant dans le questionnaire d'enquête. Elles ont ensuite « compensé » dans une troisième étape en commanditant à un étudiant en sociologie l'application de manière systématique d'un guide d'entretien approfondi à un sous-échantillon de l'enquête quantitative (voir section suivante).

Un dernier aspect sur les possibilités concrètes de dialogue concerne les différentes cultures d'explicitation de la méthode de terrain. Toute démarche de terrain, aussi qualitative et interprétative soit-elle, s'ancre dans une définition, même implicite et éventuellement sujette à redéfinition au cours même de la recherche, de ce qui va être observé. L’approche des économistes présentait un avantage pour la collaboration en ce qu'elle proposait une explicitation ex ante de ces deux aspects, à la fois pour leurs outils quantitatifs et qualitatifs : stratégie d'échantillonnage et questionnaire ou guide d'entretien. Cette explicitation a permis de constituer une base écrite de dialogue dont les anthropologues ont pu s'emparer. À l'inverse, la tradition de recherche des anthropologues du projet se prêtait moins à ce type d'exercice ex ante.

\section{Bilan}

Le pari de l'interdisciplinarité et du multi-méthodes n'a pas été sans peine. Ce qui apparaissait en première analyse comme une proximité de posture a finalement donné lieu à moins d'échanges qu'escompté entre économistes et anthropologues. On peut même se demander si ces proxi- 
mités à certains égards trompeuses n'ont pas par moments entravé le dialogue au lieu de le faciliter. Certaines pistes qui semblaient prometteuses n'ont finalement pas abouti. Par ailleurs, nous avions de toute évidence sous-estimé la lenteur du travail interdisciplinaire et de la production collective de données primaires. Mais ce pari a néanmoins permis de constituer un corpus empirique de grande qualité, permettant de déboucher sur des productions scientifiques originales et pertinentes.

Le principal acquis de la collaboration entre anthropologues et économistes concerne l'enquête quantitative. Dans les trois pays, 400 ménages ont été enquêtés suivant un protocole d'échantillonnage aléatoire visant à assurer la représentativité statistique à l'échelle de chaque petite région et un questionnaire conçu pour garantir à la fois la comparabilité de certaines données sur les trois pays, et le respect des particularités locales. Le questionnaire, initialement basé sur un modèle d'enquête d'économie agricole assez classique ${ }^{4}$, a beaucoup bénéficié d'une première phase de collecte de données réalisée par les anthropologues. Celle-ci visait à élaborer des hypothèses pertinentes et novatrices et à repérer les catégories « indigènes » de perception de la réalité, qui font sens pour les personnes et qui sont propres à un contexte donné. Elle avait pour but d'éviter de projeter des hypothèses de comportement définies a priori. Elle avait également pour but d'optimiser la fiabilité des données collectées ensuite à travers un questionnaire quantitatif en élaborant pour chaque question des possibilités de réponse adaptées, là aussi, aux réalités locales. Ont ainsi été mises en évidence dans chaque contexte la diversité des relations financières, des terminologies employées et des critères d'appréciation ainsi que la complexité des modes de gestion des familles. Cette déconstruction des catégories, qui est l'une des spécificités de l'anthropologie, a ensuite nourri l'élaboration du questionnaire. Ont également été mis en évidence le rôle déterminant des garants dans l'accès aux services financiers et les coûts multiples que cela suscite, en particulier au Mexique, ou encore le rôle fondamental des relations de clientèle, notamment en Inde, ainsi que les formes concrètes que prennent ces relations. La seconde phase de collecte de données qualitatives a ensuite été déterminante pour établir des liens

4 À l'exception d'un module innovant visant à aborder la question de la demande et du rationnement de crédit (Boucher et al., 2009). 
de causalité, des interactions entre différentes facettes de la réalité, et tenir compte de dimensions difficilement capturables par un questionnaire, comme les questions d'identité ou de pouvoir (Rao et Woolcock, 2003, pp. 182-186).

Sur le terrain mexicain, l'enquête quantitative a notamment bénéficié des résultats qualitatifs préalables sur le rôle des garants pour accéder aux crédits formels ainsi que sur l'importance du travail salarié agricole dans les systèmes de revenus des familles. L'incorporation de questions spécifiques sur les garants (fréquence, relations, importance de la réciprocité, etc.) a fourni un matériau très riche et original permettant de nourrir la réflexion sur l'enchâssement social des pratiques financières y compris dans le secteur formel. Le module sur les contrats de travail salarié agricole, complété d'entretiens semi-directifs menés ultérieurement au questionnaire, a permis de produire des connaissances novatrices sur les liens entre marché du travail et marché du crédit (à travers les arrangements sociaux et contractuels sous-jacents) et sur l'enchâssement social des marchés (Bouquet, Morvant-Roux et Rodriguez-Solis, à paraître). La réflexion s'est alimentée d'un dialogue disciplinaire entre économie néo-institutionnelle (centrée sur les caractéristiques des transactions) et de socio-économie (centrée sur la mise en ouvre concrète de la transaction et le rôle des groupes sociaux qui y contribuent). L'approche itérative et la complémentarité entre méthodes a bien fonctionné. Les entretiens semi-directifs non systématisés menés dans un premier temps avaient abouti à des hypothèses contextualisées. Les entretiens systématisés menés dans un second temps sur un sous-échantillon raisonné de 40 familles ayant participé à l'enquête quantitative ont contribué à démontrer les mécanismes de causalité à l'œuvre. L'analyse statistique, conduite de manière itérative avec la deuxième phase de terrain qualitative, a établi des ordres de grandeur et l'importance relative de certains phénomènes (en confirmant par exemple l'importance des garants, mais en minimisant celle du jonglage entre plusieurs sources de crédit), ainsi que des liens entre variables, en confortant ou en nuançant certaines hypothèses.

Sur le terrain indien, l'analyse statistique descriptive a permis de donner des ordres de grandeur à certaines tendances observées par des études de cas et de l'observation participante, par exemple le dénombrement des 
relations de clientèle (c'est-à-dire le fait que la relation de dette s'accompagne d'autres services offerts par le prêteur et/ou l'emprunteur) et le rôle significatif de la caste dans l'acte de prêt ou d'emprunt (Guérin, Roesch, Michiels et Venkatasubramanian, 2012). Inversement, le comptage nous a amenées à nuancer certaines hypothèses et à poser des questions nouvelles. Par exemple, le fait de quantifier les revenus a mis en évidence la mobilité sociale relative des basses castes (Guérin, Venkatasubramanian et Michiels, à paraître). Quant à l'analyse multivariée, elle a confirmé certaines de nos hypothèses, par exemple concernant l'absence de corrélation entre microcrédit et entrepreneuriat local et en revanche la corrélation forte entre l'appartenance de caste et de genre et la propension à entreprendre, ainsi que la nature des entreprises créées (Guérin, D’Espallier et Venkatasubramanian, à paraître). Des entretiens semi-directifs menés ultérieurement sur un échantillon raisonné ont permis d'interpréter les processus à l'œuvre, mettant en évidence le rôle de la migration de courte distance dans la mobilité sociale des basses castes et la nature des discriminations subies par les basses castes et les femmes dans la création d'entreprise.

Enfin, sur le terrain malgache, le travail de terrain et d'analyse a été mené sans apport direct de l'anthropologie, faute de ressources humaines ${ }^{5}$, même s'il a été possible de tirer parti des apports de la pluridisciplinarité menée dans les deux autres pays (notamment lors de l'adaptation du questionnaire quantitatif). Le travail d'adaptation du questionnaire au contexte local s'est également nourri de travaux qualitatifs antérieurs menés par la même équipe. Enfin, faisant suite à l'application de l'enquête quantitative, un travail qualitatif fondé sur l'application systématique d'un guide d'entretien approfondi a été mené sur 8o ménages, afin de décortiquer la complexité des modes de gestion des budgets familiaux, ainsi que les articulations entre pratiques financières formelles et informelles, en mobilisant notamment le concept de jonglage proposé par des collègues indianistes (Wampfler, Bouquet et Ralison, 2013).

5 Un anthropologue avait été sollicité mais n'a pas donné suite : démarrer un nouveau terrain sur une durée courte lui semblait très risqué. 


\section{La vie sociale d'un projet : les aléas des partenariats et des collaborations}

\subsection{Partenariats Nord-Sud}

Dans les recherches portant sur les Suds, collaborer avec nos homologues des pays étudiés est souvent considéré comme une nécessité éthique mais aussi comme une source de plus-value scientifique. Néanmoins, dans la pratique, les modalités de mise en œuvre de ces partenariats varient fortement d'un projet à l'autre et d'un contexte à l'autre, et ces dynamiques sont aussi souvent marquées par de fortes asymétries (Maselli, Lys et Schmid, 2006 ; Vidal, 2014). La Commission suisse pour les partenariats de Recherche avec les pays en Développement de l'Académie des Sciences suisse identifie deux modèles types de partenariats qui définissent deux pôles sur un continuum : le partenariat collaboratif (défini par un partage du leadership scientifique et financier) et le partenariat de délégation (défini par une relation contractuelle, le leadership scientifique et financier étant détenu par le partenaire Nord) (Maselli, Lys et Schmid, 20o6).

Le projet offre un panel intéressant pour analyser les implications en termes scientifiques (et humains) des configurations partenariales entre chercheurs du « Nord » et du «Sud» [Tableau 1]. Dans le cas du Mexique, il s'agissait d'un partenariat collaboratif relativement équilibré (stature scientifique du partenaire et budget propre). En Inde et Madagascar, il s'agissait d'un partenariat de délégation, marqué par le leadership scientifique (et financier pour l'Inde) des chercheurs « Nord » alors qu'au «Sud » les équipes locales étaient, globalement, en position de prestataires de service.

Il faut souligner la forte implication personnelle des chercheurs « Nord » sur l'ensemble des terrains, quelle que soit la configuration partenariale. En ce qui concerne l'Inde et le Mexique, les chercheurs « Nord » avaient tous vécu au moins un an dans l'un des deux pays. Pour Madagascar, les deux chercheures françaises avaient, avant le projet, réalisé de nombreux séjours dans ce pays. Les équipes « Nord » avaient donc une certaine maîtrise des contextes locaux. Les chercheurs « Nord » ont ensuite passé entre quatre et six semaines sur leurs terrains respectifs, pour participer à la production des données empiriques. 
Tableau 1. Configuration du projet

\begin{tabular}{|l|l|l|l|}
\cline { 2 - 4 } \multicolumn{1}{c|}{} & \multicolumn{1}{c|}{ Mexique } & \multicolumn{1}{c|}{ Inde } & \multicolumn{1}{c|}{ Madagascar } \\
\hline Interdisciplinarité & oui & oui & non \\
\hline Multi-méthodes & oui & oui & oui \\
\hline Partenariat Sud & collaboratif & délégation & délégation \\
\hline Post-doctorants & nord et sud & nord et sud & non \\
\hline
\end{tabular}

Source : élaboration des auteurs.

Il va de soi que l'approche partenariale par délégation est beaucoup plus simple à mettre en ouvre. En Inde comme à Madagascar, si les chercheurs «Sud » ont été pleinement associés à la construction de la recherche (y compris via les réunions annuelles qui rassemblaient l'ensemble des chercheurs des trois pays), à l'analyse puis à la valorisation, il reste que le cadre théorique et méthodologique était très largement suggéré et mâ̂trisé par les chercheurs «Nord ». Ceci a permis de contourner certaines difficultés majeures mentionnées plus haut, notamment en évitant toute confrontation de nature épistémologique. Par ailleurs, dans les deux pays, le projet s'est appuyé sur des collaborations préexistantes et s'est largement nourri d'habitudes de travail en commun. Dans les deux cas, le dispositif a fonctionné, mais a peu fait l'objet de débats, et a finalement produit plus de continuité que d'innovation.

Dans le cas du Mexique, les chercheurs « Nord » ont construit un partenariat ex nihilo avec l'équipe d'une anthropologue reconnue dans les réseaux académiques internationaux pour ses travaux sur la dette. Si la collaboration entre les deux équipes semblait prometteuse, elle a été entravée par divers obstacles, qu'il est utile de détailler.

Les principales sources de tensions voire de conflits ont été de trois ordres. La première relève des terrains respectifs des membres de l'équipe. Le travail s'est concentré sur le terrain historique d'investigation de l'équipe des anthropologues car celui-ci présentait des caractéristiques similaires aux régions des deux autres pays. À l'inverse, l'équipe mexicaine a eu accès au terrain historique des chercheuses « Nord » : ce « terrain » était constitué à la fois par une autre région, et par une institution 
de microfinance emblématique, implantée dans cette région. La question du rapport aux terrains croisés s'est néanmoins révélée problématique. Dans les deux cas, des craintes se sont manifestées sur le risque d'altérer des relations de longue date avec les acteurs locaux. L'abandon du projet d'appliquer une enquête quantitative sur le terrain historique des chercheuses « Nord » décidé pour des raisons budgétaires, a également été perçu par l'équipe «Sud » comme un désengagement unilatéral qui créait de l'asymétrie dans les contributions respectives. En définitive, et malgré l'objectif initial, ces terrains croisés n’ont pas permis de véritablement construire une base de dialogue, et ont au contraire engendré des tensions « territoriales ».

Comme nous l'avons vu dans la section précédente, le second facteur de crispation provient peut-être d'une vision trop optimiste par les chercheuses « Nord » (économistes) des proximités méthodologiques et épistémologiques avec leurs collègues «Sud » (anthropologues), alimentant chez ces dernières le sentiment d'une instrumentalisation à mauvais escient de leur discipline. Pour les économistes, ces remises en question ont été l'occasion d'une véritable démarche réflexive, même si cette démarche s'est parfois conduite à marche forcée et n'a pas véritablement fait l'objet d'une mise en commun.

Enfin, de fortes tensions sont apparues autour de l'utilisation des données produites. Cette étape de l'analyse est intervenue au terme de trois années de malentendus et d'incompréhensions, rendant problématique la possibilité de compromis. Les corpus empiriques (transcriptions d'entretiens et base de donnée quantitative) ont été mis à disposition du collectif, mais de façon parfois partielle, sous des formes non finalisées ou difficiles à mobiliser de manière autonome. Du fait même de l'implication des deux équipes dans tout ou partie du processus de collecte des données, chacun se sentait légitime pour utiliser ces corpus mais peu enclin à collaborer. Un réservoir potentiel de synergies est ainsi resté, à ce jour, largement sous-utilisé.

La collaboration sur l'analyse des données a néanmoins pu se concrétiser autour d'un projet commun de publication (Bouquet, Morvant-Roux et Rodriguez, à paraître) entre les chercheuses « Nord » et un étudiant de Master «Sud », qui avait assuré la conduite d'entretiens approfondis après 
le passage du questionnaire quantitatif, et contribué à l'analyse qualitative. Mais cet aboutissement a finalement repris la forme d'un schéma de "délégation », comme dans les deux autres pays, et il a en outre suscité des tensions « territoriales » supplémentaires, autour de la question de l'implication de cet étudiant. De son côté, l'équipe «Sud » a développé ses propres analyses, et a noué des collaborations fructueuses avec certains membres du projet sur plusieurs ouvrages collectifs (Guérin, Morvant-Roux et Villarreal (dir.), 2013 ; Angulo et Villarreal (dir.), 2013; Villarreal (dir.), 2014).

Ces tensions auraient pu se présenter également sous une configuration partenariale Nord-Nord. Cependant, la configuration Nord-Sud a probablement joué en notre défaveur dans la mesure où les tensions se sont alimentées de problèmes récurrents de communication à distance et dans plusieurs langues, créant un effet cumulatif. Les dysfonctionnements du bailleur associé à l'ANR (six mois de retard dans le versement de la première tranche de financement au partenaire mexicain) ont également pénalisé dans sa phase initiale la construction d'une relation de confiance.

\subsection{Chercheurs statutaires, chercheurs contractuels}

Un aspect important de la vie sociale du projet tient à la position particulière des cinq post-doctorants par rapport aux chercheurs statutaires. Le statut des chercheurs précaires, en recherche de poste par définition, entre particulièrement en résonance avec les enjeux d'un projet de recherche impliquant plusieurs disciplines et plusieurs méthodes, en raison de la prise de risque que ce type de démarche implique, à la fois en termes de positionnement académique et en termes de temporalités. Les post-doctorants devaient manœuvrer entre des injonctions contradictoires, valorisant dans les discours l'innovation scientifique, dont l'interdisciplinarité, et privilégiant dans les faits du marché de l'emploi académique les profils standard, avec un ancrage disciplinaire clair et des publications répondant aux canons de la discipline considérée. En termes de temporalités, la contradiction était également très forte. Les post-doctorants n'étaient officiellement impliqués (i.e. payés) que sur une période partielle du projet. Il était indispensable de publier dans ce laps de temps court pour pouvoir espérer valoriser cette expérience professionnelle, ce qui était difficilement compatible avec le caractère chronophage du processus de production de 
données. Si le bilan du projet en matière d'insertion professionnelle des post-doctorants n'est pas déshonorant (quatre postes statutaires, dont deux au Sud, et un poste contractuel), le bilan en matière de contribution au projet est resté, pour la plupart d'entre eux, en deçà de leur potentiel, et des pistes prometteuses en matière d'interdisciplinarité et de multi-méthodes sont restées en friche.

\section{Enseignements}

Le dialogue entre disciplines et la combinaison des méthodes a été source d'innovation et de créativité, mais aussi source de frictions, probablement parce que nous voulions trop faire ensemble. Définir dès le départ ce que chacun entendait par « quantitatif » et « qualitatif » nous aurait évité bien des déconvenues. Les clivages épistémologiques sous-jacents à l'usage multiforme de ces termes (qu'est-ce qui est observable et à quel mode d'administration de la preuve a-t-on recours ?), identifiés au fil des échanges, auraient mérité d'être explicités dès le départ. Cela aurait supposé une démarche collective de réflexivité, qui ne va pas de soi, car elle demande entre autres une culture minimale commune en épistémologie. Ces clivages n'étaient pas irréductibles mais circonscrivaient plus étroitement les possibilités de collaboration. Il aurait été utile d'identifier et de redéfinir (au fur et à mesure que la recherche évoluait) les thèmes mais aussi les étapes possibles de collaboration : certaines phases de collecte des données peuvent être collectives, mais l'analyse, l'écriture et la valorisation, pour être véritablement collectives, supposent d'être très en phase, y compris au sein de la même discipline. Ménager davantage d'espaces d'autonomie permettant d'avancer en parallèle aurait peut-être pu apaiser certaines tensions, au sein des partenariats comme entre chercheurs statutaires et contractuels.

Un autre constat, beaucoup plus classique, a trait à la temporalité. Un projet qui combine les méthodes de manière itérative (au sens où l'approche compréhensive précède et succède la collecte puis l'analyse statistiques) peut difficilement se dérouler sereinement sur une période de trois ans. Chaque étape suppose une longue phase de préparation puis de maturation, a fortiori lorsqu'elle repose sur un partenariat. Nous avions également sous-estimé le temps nécessaire pour créer du lien et un 
sentiment d'appartenance à un projet collectif. Nous l'avions d'autant plus sous-estimé que nombre d'entre nous ne se connaissaient pas et que notre équipe était très éclatée géographiquement. De toute évidence, nous n'avons pas consacré assez de moyens aux réunions collectives. Il est certainement irréaliste de prétendre « chercher ensemble » sans passer beaucoup de temps ensemble, à plus forte raison lorsqu'il s'agit de défricher des thèmes ou des méthodes.

À ce jour, les économistes du projet sont parvenues à mettre à profit la composante interdisciplinaire et multi-méthodes, mais l'inverse n'a pour l'instant pas fonctionné (ce constat d'asymétrie rejoint celui de Bardhan et Ray, 2008). Le projet avait été conçu initialement par des économistes. Nous avions clairement identifié les points sur lesquels les anthropologues pouvaient nous éclairer, tandis que ces derniers, et en dépit d'un intérêt qui semblait réel pour notre démarche et notre souci de quantification, sont toujours restés flous sur l'articulation possible avec leurs propres méthodes. Une construction conjointe du projet aurait peut-être permis d'aplanir ce type de difficultés, mais cela aurait supposé un pas de temps peu compatible avec le délai serré de l'appel d'offre. Il est par ailleurs, probable que l'asymétrie des techniques ait joué un rôle. Il est relativement plus facile pour les économistes de s'approprier les éléments d'une analyse compréhensive (même si c'est de manière plus ou moins efficace et rigoureuse). En revanche l'inverse, compte tenu des compétences purement techniques que suppose l'analyse statistique, est plus difficile.

Concernant les partenariats «Suds », deux leçons se dégagent. Lorsque la collecte de données suppose une infrastructure lourde (enquêteurs, personnes ressources, etc.), fonctionner avec des équipes locales est incontournable, mais prétendre construire systématiquement des partenariats collaboratifs sur cette base est certainement illusoire. Mieux vaut clairement afficher un fonctionnement sous la forme de la délégation et de la prestation de services, qui n'a rien de honteux à partir du moment où il est clairement assumé. Quant aux partenariats collaboratifs, ils supposent un partage du leadership scientifique (et financier) ; or le premier de ces partenariats a peu de chances de se concrétiser s'il n'intervient pas dès la conception du projet. 
Une dernière leçon, probablement essentielle, porte sur le pragmatisme que suppose un projet de ce type. Il faut savoir accepter les revers et les déceptions et les considérer comme partie prenante de la démarche. Dans la recherche comme ailleurs, la créativité et l'innovation supposent de la prise de risque. La collaboration, quelle qu'elle soit, et puisqu'elle implique des relations humaines, est nécessairement soumise à la contingence et à l’impondérable.

\section{Conclusion}

À partir d'un cas singulier, ce texte avait pour objet d'ouvrir la boîte noire de la collaboration scientifique. Nos résultats, résumés ici brièvement, confirment les synergies et complémentarités entre approches statistiques et compréhensives, entre économie et anthropologie, qu'il s'agisse de mieux comprendre des chaînes de causalité, de questionner des catégories ou encore d'éviter la surinterprétation d'observations localisées. Malgré ou peut-être grâce à son déroulement parfois cahotant, notre projet a apporté une contribution à la production de connaissances scientifiques sur plusieurs plans (King et al., 1994, pp. 14-28) : en posant de meilleures questions, en améliorant la qualité des données et en améliorant l'usage fait des données ${ }^{6}$.

Nombre de méthodes mixtes échouent ou donnent des résultats décevants car elles sous-estiment le clivage épistémologique qui oppose approche statistique et approche compréhensive quant aux modes d'administration de la preuve (Kanbur et Shaffer, 2007). Elles sous-estiment également l'exigence de respect mutuel, condition essentielle d'un dialogue constructif (Bardhan et Ray, 2008, et enfin, les moyens, humains et financiers, que cela suppose. Nous concernant, ces trois aspects ont certainement constitué des points d'achoppement. Notre proximité était à certains égards trompeuse, et notre intérêt commun pour certains concepts masquait parfois de profondes divergences. Celles-ci ne sont pas irréductibles lorsque l'on se contente de dialoguer, mais peuvent le devenir lorsqu'il s'agit réellement de « chercher ensemble ». Pour autant, il ne s'agit pas de

6 King et al., (1994) mentionnent une quatrième catégorie : amélioration de la théorie. Notre projet n'affichait pas une telle ambition. 
renoncer aux ambitions de l'interdisciplinarité, des méthodes mixtes et du partenariat, mais d'être conscient des écueils pour pouvoir mieux les anticiper et les gérer à l'avenir.

\section{AUTEURS}

\section{Isabelle Guérin}

Directrice de recherches en socio-économie à l'IRD, UMR 245 Cessma.

\section{Emmanuelle Bouquet}

Chercheure en économie au Cirad, UMR Moisa.

\section{Solène Morvant-Roux}

Maître-assistante en socio-économie à l'Institut de démographie et de socio-économie de l'université de Genève (Suisse). 


\section{BIBLIOGRAPHIE}

Angulo L., Villarreal M., (dir.), 2013, Las microfinanzas en los intersticios del desarrollo. Cálculos, normatividades y malabarismos, Mexico, UPN/FOJAL/ CIESAS/JAL.

Beaud S., Weber F., 2003, Guide de l'enquête de terrain, Paris, La Découverte.

Bardhan P., Ray I., (dir.), 2008, The contested commons: conversations between economists and anthropologists, Oxford, Blackwell Publishing.

Boucher S., Guirkinger C., Trivelli C., 2009, "Direct Elicitation of Credit Constraints: Conceptual and Practical Issues with an Application to Peruvian Agriculture", Economic Development et Cultural Change, vol. 57, $\mathrm{n}^{\circ} 4$, pp. 609-640.

Bouquet E., Morvant-Roux S., Rodriguez-Solis G., à paraître, "Agricultural workers, credit rationing and family networks in rural Mexico", Journal of Development Studies.

Copans, J., 2010, Un demi-siècle d'africanisme africain. Terrains, acteurs et enjeux des sciences sociales en Afrique indépendante, Paris, Karthala.

Couty Ph., Winter G., 1983, Qualitatif et quantitatif : deux modes d'investigation complémentaires. Réflexions à partir des recherches de I'ORSTOM en milieu rural africain, Paris, ORSTOM.

Guérin I., Michiels S., Venkatasubramanian G., à paraître, "Labour in contemporary South India", in Heyer J., Harriss-White B., (dir.), Capitalism in Development, London, Routledge.

Guérin I., D'Espallier B., Venkatasubramanian G., à paraître, "The social regulation of markets. Why microcredit fails in promoting jobs in rural South-India?", Development and Change.

Guérin I., Roesch M., Michiels S., Venkatasubramanian G., 2012, "Dettes, protections et solidarités en Inde du Sud ", Économie et Sociétés, vol. XLVI, $n^{\circ} 2$, p. 385-413.

Guérin I., Morvant-Roux S., Villarreal M., (dir.), 2013, Microfinance, debt and Over-indebtedness. Juggling with money, London, Routledge.

Guérin I., Lapenu C., Doligez F., (dir.), 2009, La microfinance est-elle socialement responsable? $\mathrm{n}^{\circ}$ spécial, Revue Tiers Monde, n 197.

Kanbur R., Shaffer P., (dir.), 2007, Experiences of Combining Qualitative and Quantitative Approaches in Poverty Analysis. Numéro special, World Development, vol. $35, n^{\circ} 2$.

King G., Keohane R., Verba S., 1994, Designing Social Inquiry. Scientific inference in qualitative research. Princeton, New Jersey, Princeton University Press.

Maselli D., Lys J-A., Schmid J., 2006, Améliorer l'impact des partenariats de scientifiques. Commission suisse pour le partenariat scientifique avec les pays en développement, KFPE, Berne, Geographica Bernensia.

Picherit D., 2013, "Protection and over-indebtedness in rural South India: the case of labour migrants in Andhra Pradesh", in Guérin et al. (dir.) Microfinance, debt and Over-indebtedness. Juggling with money, London, Routledge, pp. 151-170.

Rao V., Woolcock M., 2003, "Integrating qualitative and quantitative approaches 
in program evaluation", In Bank T. W., (dir.), The impact of economic policies on poverty and income distribution: evaluation techniques and tools, Washington D.C., World Bank and Oxford University Press, pp. 165-190.

Roesch M., Héliès O., 2007, "La microfinance, outil de gestion du risque ou de mise en danger par sur-endettement ? Le cas de I'Inde du Sud ", Autrepart, $n^{\circ} 44$, p. 119-140.

Vidal L., (dir.), 2014, Expériences du partenariat au Sud. Le regard des sciences sociales, Marseille, Éditions de l'IRD.

Villarreal M., Guérin I., Kumar S., 2013, Carola and Saraswathi: Juggling wealth in India and in Mexico, IMFTI Working Paper.
Villarreal M., (dir.), 2014, Las deuda de los oprimidos en el imperio de la liquidez, Numéro spécial, Desacatos, nº 44.

Villarreal M., 2009, "Erratic Hopes and Inconsistent Expectations for Mexican Rural Women: A Critique of Economic Thinking on Alternatives to Poverty", in Bhavnani K., Foran J., Kurian P. A., Munshi D., (dir.), On the Edges of Development: Cultural Interventions, New York and Oxon, Routledge.

Wampfler B., Bouquet E., Ralison E., 2013, "Does juggling mean struggling? Insights into the diversity and the complexity of rural households financial practices in Madagascar", in Guérin I., Morvant-Roux S., Villareal M., (dir.), Microfinance, debt and over-indebtedness. Juggling with money, Oxford, Routledge, pp. 211-231. 